\title{
Airborne GPS kinematic positioning and its application to oceanographic mapping
}

\author{
Shaowei Han and Chris Rizos \\ School of Geomatic Engineering, The University of New South Wales, Sydney NSW 2052, Australia
}

(Received January 16, 2000; Revised August 11, 2000; Accepted August 14, 2000)

\begin{abstract}
Precise, long-range, airborne GPS kinematic positioning requires the use of carrier phase measurements, the data processing of which suffers from the technical challenges of "on-the-fly" ambiguity resolution and cycle slip repair. In this paper the authors describe how the combination of an 'ambiguity recovery' technique and a 'linear bias correction' method has been used to support oceanographic mapping in Australian waters, together with the augmentation from the Laser Airborne Depth Sounder (LADS) system. Two experiments, carried out on the 4th December 1997 in the Torres Strait between Papua New Guinea and Australia, and on the 20 May 1998 at Lake Argyle in Australia, were analysed. The results indicate that the topography of the water surface can be obtained with sub-decimetre accuracy, with a spatial resolution of a few metres. The main errors are attributable to multipath interference of the GPS signals at the antennas from the aircraft surface.
\end{abstract}

\section{Introduction}

The Laser Airborne Depth Sounder (LADS) system was developed to permit airborne laser bathymetry, by the collection of accurate, high-density bathymetric data using twocolour laser light pulsed from an aircraft which provides distances from aircraft to both the instantaneous sea surface and the bottom of the sea (Sinclair, 1999). However, the accuracy of the bathymetric depths will be affected by the sea surface variations caused by sea swell and local tide because these depths are referenced to the instantaneous sea surface. If the geodetic height of the aircraft can be provided by airborne GPS kinematic positioning, the sea surface heights and bathymetric chart depths can then be determined. The challenge was to test the feasibility and accuracy of GPS kinematic positioning techniques for long-range airborne applications such as laser bathymetry. A promising technique, involving the use of an 'ambiguity recovery' algorithm, has been successfully used for previous long-range, kinematic positioning tests (Han, 1997). One drawback is that this algorithm requires that the integer ambiguities be initially resolved to their correct values. However, for longrange and long-duration (many hours) airborne GPS kinematic positioning it is almost impossible to maintain the integer ambiguity values, and of course "on-the-fly" ambiguity re-initialisation is typically not an option. Fortunately for oceanographic mapping, the LADS technology can provide measurements of the distance between the LADS-bearing aircraft and the ocean surface. If the average ocean surface over a few tens of kilometres is assumed to be a constant, this additional constraint can be used to correct the linear biases caused by incorrect ambiguities, and sub-decimetre level height accuracy for the ocean surface can still be derived (Han and Rizos, 1996). This idea has been used for

Copy right $($ C The Society of Geomagnetism and Earth, Planetary and Space Sciences (SGEPSS); The Seismological Society of Japan; The Volcanological Society of Japan; The Geodetic Society of Japan; The Japanese Society for Planetary Sciences. aerial photogrammetry (Ackermann and Schade, 1993).

The combination of these two techniques has been used for oceanographic mapping applications, under the assumption that: (1) the average sea surface height with respect to the ellipsoid is a constant over a small region or linear with respect to longitude, and; (2) the distance between the aircraft and the sea surface is known. The first assumption is reasonably valid because the local tide and sea swell contain mainly high frequency components, and any possible low frequency components due to the geoid errors can be corrected using existing geopotential models. In the case of the second assumption, the Laser Airborne Depth Sounder was used to directly measure the distance between the aircraft and the sea surface.

Two experiments were conducted to firstly assess the suitability of state-of-the-art, geodetic-quality GPS receivers for long-range, carrier phase-based positioning in support of the Royal Australian Navy's Laser LADS system. Secondly the utility and accuracy of the kinematic GPS technique to determine aircraft height to a high accuracy, over very long baselines were tested, which would permit the determination of the sea surface profile during LADS surveys. The first experiment was conducted over Torres Strait, north of Cape York, between Australia and Papua New Guinea in December 1997. Three GPS receivers shared one antenna on the aircraft, and two receivers were deployed at each of the reference sites, Cairns and Thursday Island. The accuracy of the height component is consistent, being within $2 \mathrm{~cm}(1 \sigma)$. However, any significant multipath cannot be revealed from data comparisons because all the GPS data suffered from the same multipath from the aircraft. The second experiment was carried out at Lake Argyle, near Kununurra, on 20 May 1998. Two NovAtel receivers with two antennas were installed on the aircraft with separation of 1.8 metres, while at the reference site at the Darwin Royal Australian Airforce Base (about $480 \mathrm{~km}$ away), the third NovAtel receiver was 
set up. Two independent trajectories for the two antennas on the aircraft were then derived, which could be used to analyse the multipath effect on the positioning accuracy.

The results indicate that the surface topography of the ocean and the lake can be obtained with sub-decimetre accuracy, with the main errors being attributable to multipath from the aircraft. A comparison of experimental results and the result derived AUSGEOID98 has been attempted.

\section{Oceanographic Mapping Using GPS and LADS Systems}

The LADS system uses the infrared pulse from a Nd:YAG laser to determine the aircraft-to-sea-surface distance, at a sampling rate of $168 \mathrm{~Hz}$. These data can be combined with the GPS-derived heights to determine the required nadir sea surface profile. The GPS trajectory heights $\left(H_{\mathrm{GPS}}\right)$ are referenced to the WGS84 ellipsoid (Fig. 1). The LADS laser heights $\left(H_{\text {Laser }}\right)$ are referred to the nadir sea surface, which nominally follows the local geoid (geoid heights $N$ ), except that it is lifted by the local tide $\left(H_{\text {Tide }}\right)$. The relationship can be represented as follows (Fig. 1):

$$
H_{\mathrm{GPS}}-H_{\text {Laser }}=H_{\text {Swell }}+H_{\text {Tide }}+N
$$

where $H_{\text {Swell }}$ is the sea swell height, whose mean value can be assumed to be zero over an area of few tens of kilometres across.

In order to remove the linear biases caused by incorrect integer ambiguity estimation, geoid heights were computed and interpolated from the AUSGEOID98 model (1 second gridded data) using the AUSLIG interpolation software Winter (Windows Interpolation software, http: // www. auslig.gov.au/geodesy/ausgeoid). After removing the geoid height values, a linear fitting has been applied to remove the constant and slope term in $H_{\mathrm{GPS}}-H_{\mathrm{Laser}}$. This means that the constant term and the slope term in $H_{\text {Swell }}+H_{\text {Tide }}$ (if there are any) over each run have also been removed at the same time, although it could be assumed to be very small and could be ignored for this application. The last step is to restore the geoid height values so that the surface heights can be determined.

For this procedure the absolute accuracy of the aircraft trajectory is not important and only the relative trajectory height

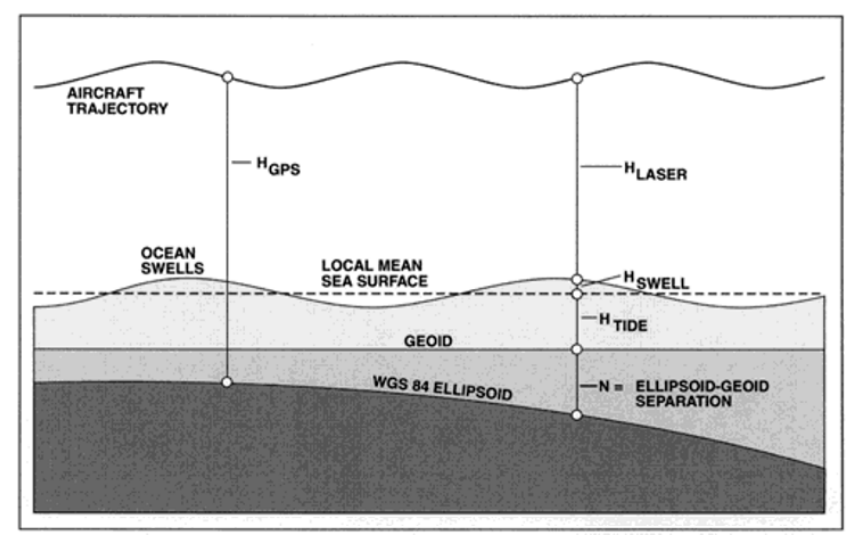

Fig. 1. Sea surface determination using GPS and LADS. is required, provided it is consistent along the duration of each run. This opens up the possibility of GPS kinematic positioning over baseline lengths of up to $1000 \mathrm{~km}$ with an accuracy of a decimetre or better, using the combination of the 'ambiguity recovery' technique and the 'linear bias correction' method.

\section{Data Processing for Long-Range GPS Kinematic Positioning}

The aircraft was flown for about 10 hours for each experiment and over many hundreds of kilometres in relation to the reference receivers. Although the integer ambiguities could be fixed when the aircraft was on the airport runway, the integer ambiguities could not be maintained for the whole flight due to many data interruptions and newly rising satellites. Therefore, the procedure for data processing for the experiments involved two steps: the 'ambiguity recovery' technique (Han, 1997), and the correction procedure for linear biases caused by incorrectly resolved ambiguities (Han and Rizos, 1996).

\subsection{Ambiguity recovery technique}

The ambiguity recovery technique resolves the cycle slip(s) using a combination of cycle slip repair and ambiguity resolution procedures (Han, 1997). This technique effectively involves "relative" ambiguity determination, rather than "absolute" ambiguity resolution. The concern is only with the biases affecting the carrier phase observations which change with time. Two steps have been suggested in this procedure and the cycle slip repair procedure was used, rather than ambiguity resolution procedure.

The cycle slip repair procedure uses a combination of precise pseudo-range and carrier phase observations, which can eliminate the receiver and satellite clock biases, and the user receiver's dynamics. The remaining biases, due to orbit error, the ionosphere, the troposphere and the static receiver's multipath change quite smoothly and can be modelled by a linear function for a short period of time. The selected widelane (wavelength $0.86 \mathrm{~m}$ ), and the other data combination with wavelength $14.65 \mathrm{~m}$ used for cycle slip detection, minimise the effect of the noise from the pseudo-range data. The other feature is the odd-even relationship between cycle slips on the widelane and the second combination (see Han, 1997, for mathematical details), which has the effect of doubling the wavelength of one combination if the cycle slip on the other one can be determined. If the data gap is quite short (less than 1 minute) cycle slips in the carrier phase observations can normally be detected in the one-way receiver-satellite data.

After all cycle slips have been removed, ambiguities can be estimated using the pseudo-range data. These are typically biased at the decimetre level, causing ambiguity resolution estimates biased up to a few cycles. When these incorrect ambiguity values are fixed and the resultant 'carrier-range' data are used to estimate the aircraft trajectory, the trajectory can be subsequently biased and the correction procedure discussed below becomes necessary.

\subsection{Correction procedure for the biased trajectory}

The direction from a GPS antenna to a satellite changes relatively slowly, hence the direction cosines also change slowly, in an approximately linear manner over a short pe- 
riod. Making use of this property, it can be shown that the incorrect initial ambiguity estimates bias the baseline vector approximately linearly with time, provided that the ambiguity error is small (a few cycles), and the time span is short (up to about half an hour).

Here the emphasis is on the relative height of the aircraft trajectory, as the sea surface height can be derived using the aircraft height above the sea surface measured by the Laser Airborne Depth Sounder. It is obvious that the sea surface's orthometric height derived in this way will be linearly biased due to any ambiguity resolution error. Fortunately these linear biases of the sea surface can be removed if the average sea surface is assumed to be zero. The details of the procedure can be found in Han et al. (1999).

The laser heights are available at $168 \mathrm{~Hz}$, whereas the trajectory heights are at the receiver rate, which for Ashtech receiver was $5 \mathrm{~Hz}$, for the Leica receiver was $10 \mathrm{~Hz}$, and for the NovAtel Millennium receiver was $4 \mathrm{~Hz}$. A cubic spline interpolator was used to derive the trajectory values at $168 \mathrm{~Hz}$, which were then combined with the laser heights as explained above.

\section{Experiments}

\subsection{Torres Strait experiment}

The first experiment was conducted on the 4 December 1997. The LADS survey area for this experiment was located over the Torres Strait, north of Cape York, between Australia and Papua New Guinea. The primary reference stations (NovAtel and Leica receivers) were located at Cairns Airport where the LADS F27 aircraft was based, about 900 $\mathrm{km}$ south of the survey area. The secondary (temporary) reference stations (NovAtel and Ashtech) were located on Thursday Island in the Torres Strait, about $100 \mathrm{~km}$ south of the survey area (Fig. 2). Three receivers (Ashtech, Leica and NovAtel receivers) were installed in the aircraft and fed by the same antenna using a signal splitter (the left figure in Fig. 3). In a single flight sortie, LADS travelled from Cairns to the survey area, where it remained on-station for 2.5 hours, collecting swaths of water depth data before returning to Cairns. All GPS receivers started to collect data at about 9:30 am (local time, $10 \mathrm{hr}$ ahead of UT) and ended at 7:30 pm. For each sortie the aircraft took off from Cairns Airport and travelled about $900 \mathrm{~km}$ to the Torres Strait area, then returned after the survey was completed.

The trajectories for six runs in the West-East direction and two runs in the North-South direction are shown in Fig. 4. The difference between the GPS height and the LADS range are sea swell, local tide, geoid height and the linear bias caused by incorrect ambiguity resolution. The geoid heights are computed and interpolated using Winter software based on the AUSGEOID98 model ( 1 second gridded data). After removing the geoid height, a linear fitting procedure was used to remove the remaining linear bias term. The remaining information contains the sea swell, local tide and measurement noise with zero-mean, which is plotted in Fig. 5 (with the lower four curves for Run 1 derived from four GPS data sets). The results of Ashtech_TIS means the results are derived from the Ashtech aircraft receiver and the Ashtech reference receiver on Thursday Island. The results of Leica_CNS $+0.5 \mathrm{~m}$ are derived using the Leica

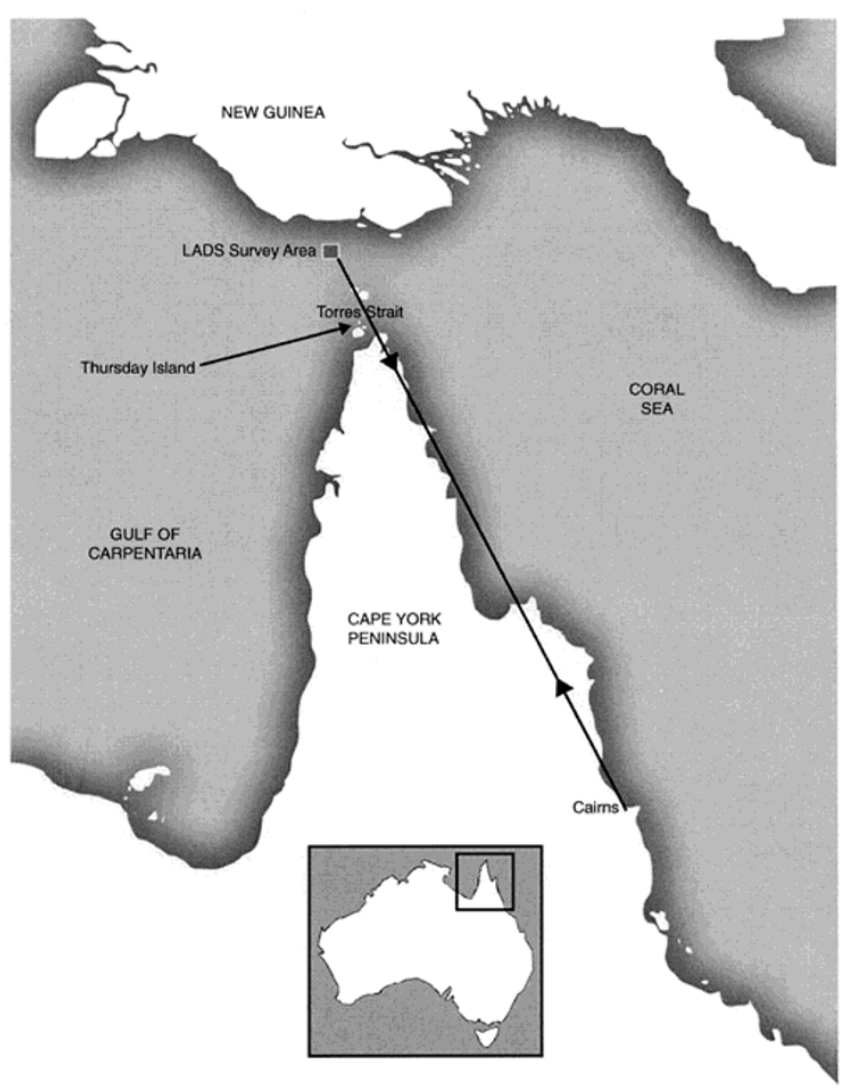

Fig. 2. Location of the Torres Strait experiment off Queensland, Australia.

receivers in the aircraft and at Cairns with $0.5 \mathrm{~m}$ shift in order to provide separation in the figure. The results of NovAtel_CNS $+1.0 \mathrm{~m}$ are derived using the NovAtel aircraft receiver and NovAtel receiver at Cairns with $1.0 \mathrm{~m}$ shift. The results of NovAtel_TIS $+1.5 \mathrm{~m}$ are derived using the NovAtel receivers in the aircraft and on Thursday Island, with a shift of $1.5 \mathrm{~m}$. The difference between the different data sets are also presented in Fig. 5, represented by the upper three curves. The top curve is the difference between the two NovAtel results, using the Cairns $(900 \mathrm{~km})$ and Thursday Island $(125 \mathrm{~km})$ reference receivers. The second curve is the difference between the NovAtel (Thursday Island reference receiver) and the Leica (Cairns) results. The third curve is the difference between the NovAtel (Thursday Island reference receiver) and the Ashtech (Thursday Island) results. The maximum difference is at the $6 \mathrm{~cm}$ level, and the standard deviations for the top, the second and the third curves are $1.9 \mathrm{~cm}, 2.3 \mathrm{~cm}$ and $1.9 \mathrm{~cm}$ respectively.

If the geoid height derived from the AUSGEOID98 model is restored back, the sea surface height can be derived. As an example, Fig. 6 illustrates the results for sea surface heights derived by the NovAtel receivers (Thursday Island as reference site) for the six runs. The smooth curves are geoid heights derived from AUSGEOID98. The results derived by the other three data sets are similar to those shown in Fig. 5 for Run 1 .

It can be seen that the trajectories derived from different GPS data sets are consistent with each other. However, the multipath disturbance from the aircraft cannot be estimated 

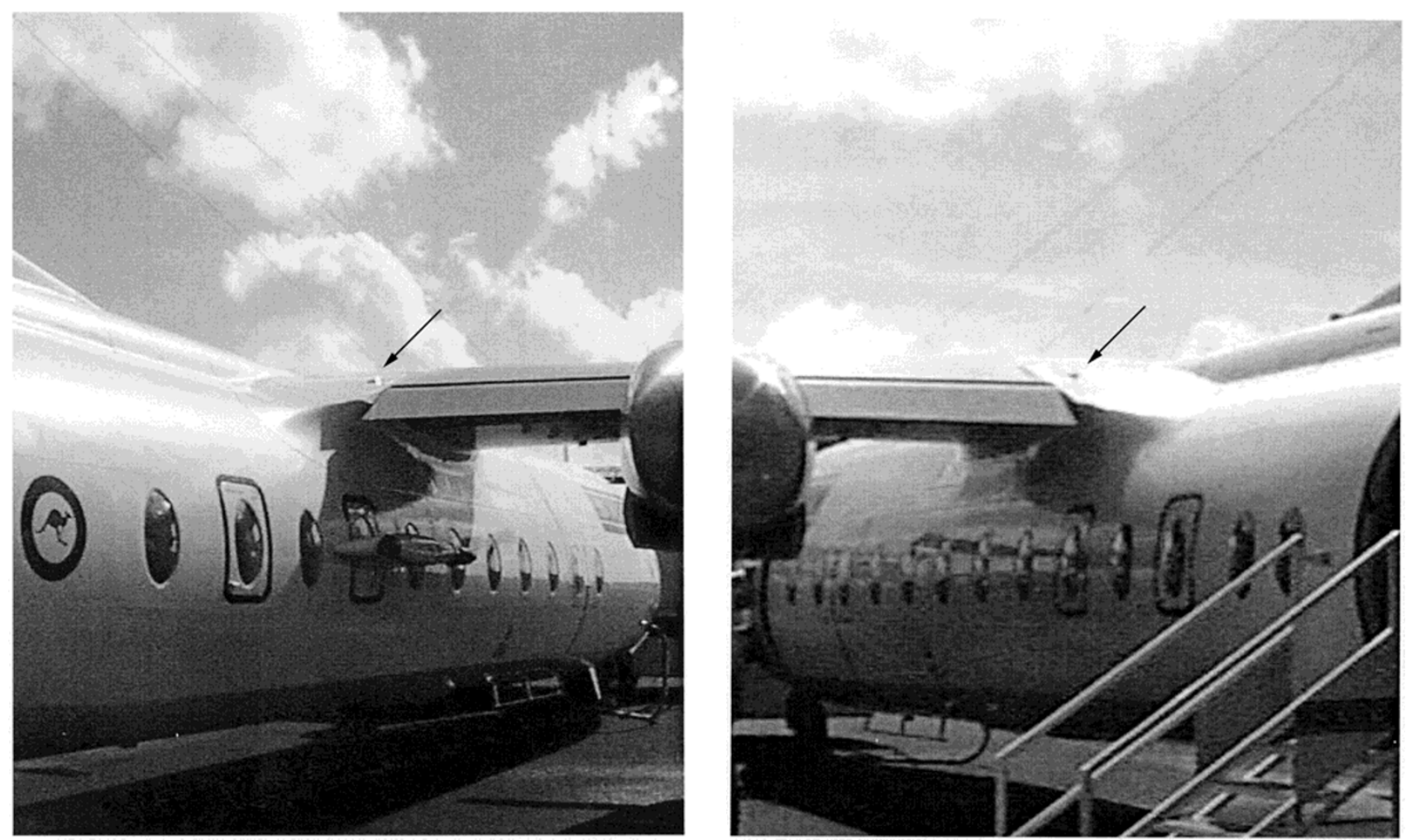

Fig. 3. The small "bumps" in the middle of both photos, on the upper surface of both wings, are GPS antennas (NovAtel 512). The horizon from the antenna is 12 degrees up to the fuselage top and 10 degrees up to the wing top. The antenna on the left figure was used for the Torres Strait experiment, and both antennas were used for the Lake Argyle experiment.

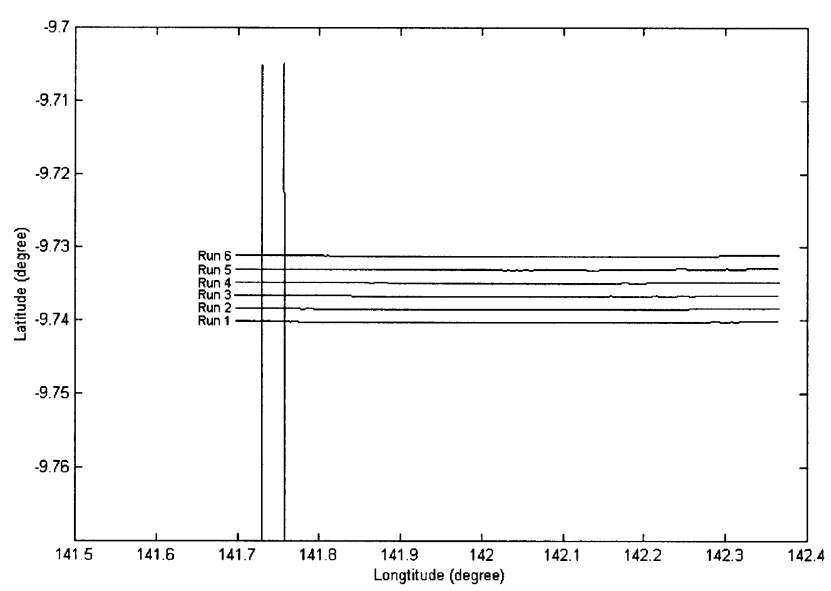

Fig. 4. Six runs in the West-East direction and two runs in the North-South direction (Day 338).

because the same antenna was used for all aircraft receivers. Therefore, an analysis of multipath must be carried out in another way. Figure 7 shows the multipath effects on the double-differenced L1 and L2 carrier phase measurements (satellite PRNs 5 and 9) for the two NovAtel receivers, at the reference site and on the aircraft. The multipath effect on the double-differenced carrier phase measurements are mainly attributed to the multipath at the aircraft antenna (the multipath at the reference receivers is very small, as verified from the residuals of the baseline between the reference receivers).

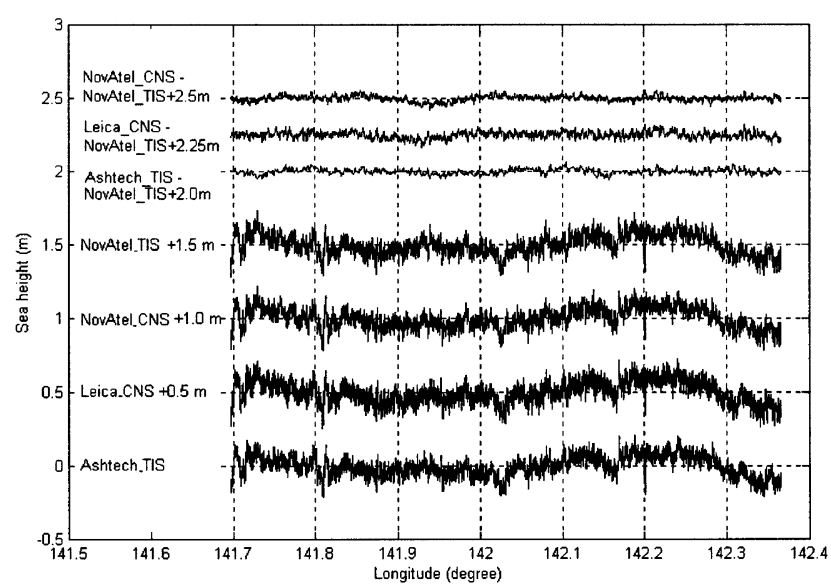

Fig. 5. Sea swell heights and variations of local tide and geoid for Ashtech receivers (TIS-Aircraft), Leica receivers (CNS-Aircraft) and NovAtel receivers (TIS-Aircraft and CNS-Aircraft) for Run 1 at Torres Strait.

It can be seen that the multipath effects are significant.

The results indicate that all three brands of GPS receivers can be used for long-range, carrier phase-based, airborne applications with high data rate output. The 'ambiguity recovery' technique in combination with the 'linear bias correction' procedure over short periods (typically half an hour) can be successfully used. The discrepancies among the heights derived from the different data sets are at the centimetre level. The geopotential model is consistent with the sea surface. The multipath effect is significant for the aircraft 

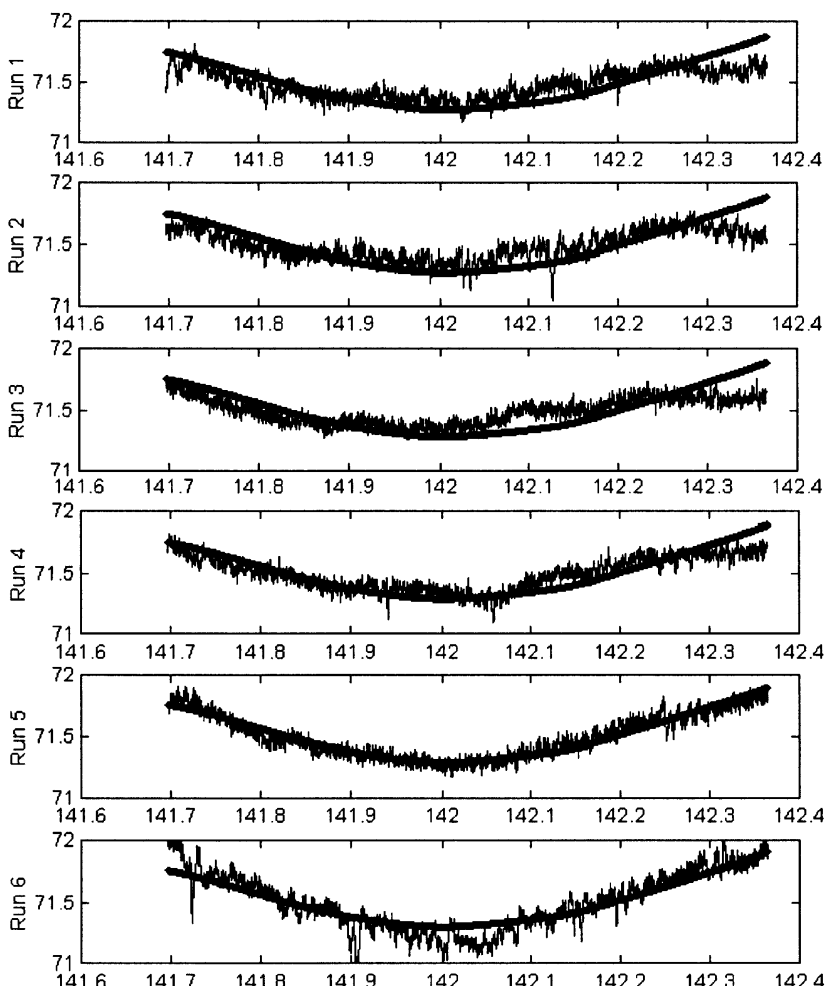

Fig. 6. Sea surface heights derived from GPS and LADS (noise curves) and geoid heights from AUSGEOID98 (smooth curves).
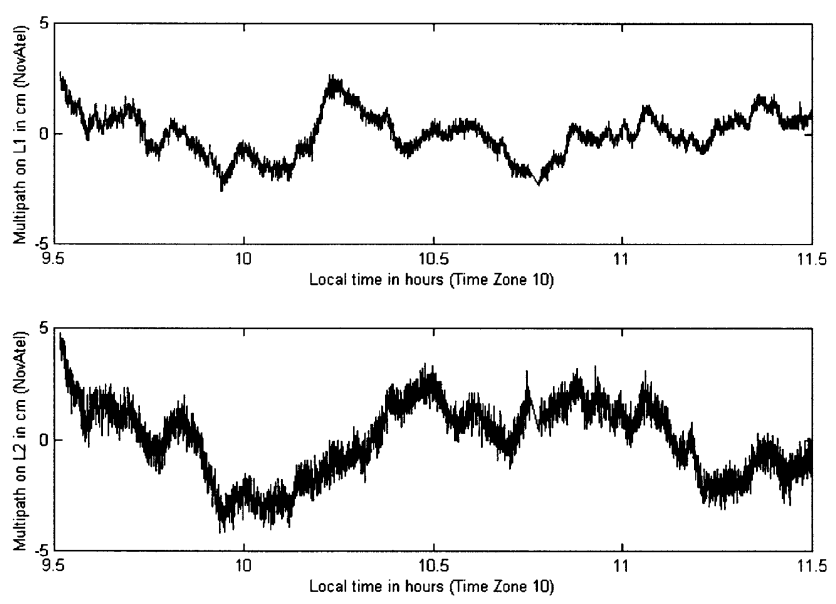

Fig. 7. Multipath effects on L1 and L2 double-differenced carrier phase of PRNs 5 and 9 for the NovAtel receiver at the reference site and the NovAtel receiver at the aircraft.

environment but cannot be revealed from single antenna experiments. Therefore two GPS antennas were installed on the aircraft for the second experiment.

\subsection{Lake Argyle experiment}

Although the technique of oceanographic mapping using a combination of GPS and LADS was validated during the previous experiment, the accuracy could not be assessed due to the use of a single antenna on the aircraft. The second experiment was carried out at Lake Argyle, near Kununurra, on 20 May 1998. Two NovAtel antennas were installed on
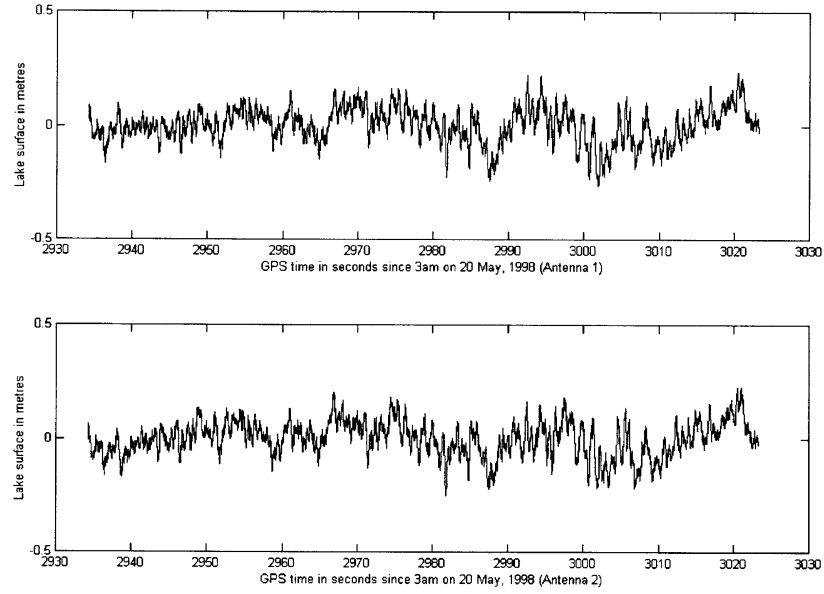

Fig. 8. Lake surface heights derived from GPS and LADS for Run 1 (the upper figure is the result derived from Antenna 1, shown on the left side of Fig. 3, and the lower figure is the result derived from antenna 2, shown on the right side of Fig. 3).
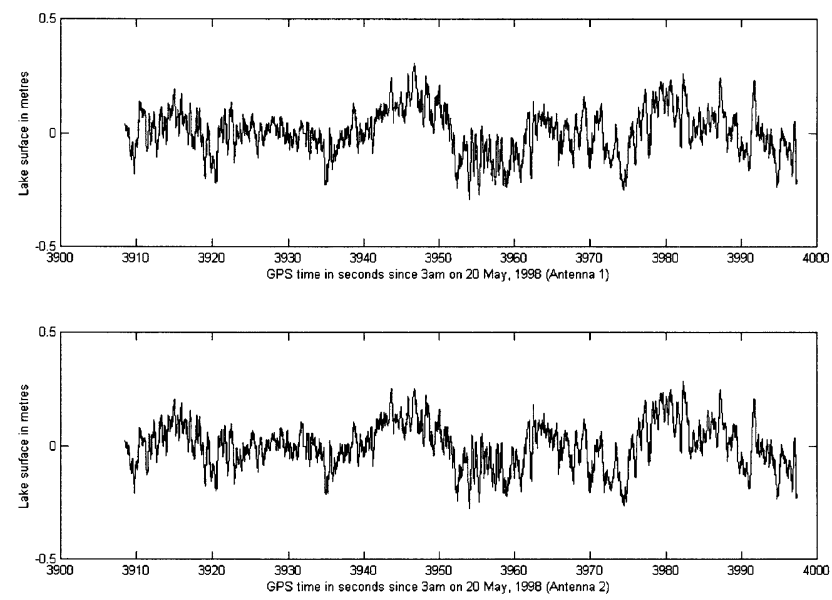

Fig. 9. Lake surface heights derived from GPS and LADS for Run 2 (the upper figure is the result derived from Antenna 1, and the lower fugure is the result derived from antenna 2).

the aircraft, with a separation of about 1.8 metres, and all GPS receivers logged at $4 \mathrm{~Hz}$. The LADS system measured the distance between the aircraft and the lake surface. The experiment permitted the derivation of two independent trajectories for the two antennas on the aircraft, and the analysis of the impact of multipath on the results. The distance from the reference receiver at Darwin to the LADS survey area was about $480 \mathrm{~km}$.

The same procedure was used to derive the lake surface heights. These are plotted in Figs. 8 and 9. It can be seen that the results are again consistent. The variations of the lake surface may be due to lake swell, the LADS laser height noise and GPS multpath. However, the multpath effects on the results are not clear from these figures. The distance between the two antennas was computed, and is plotted in Fig. 10. It can clearly be seen that the multipath effect can reach $10 \mathrm{~cm}$ !

The derived lake surface heights have quite large varia- 

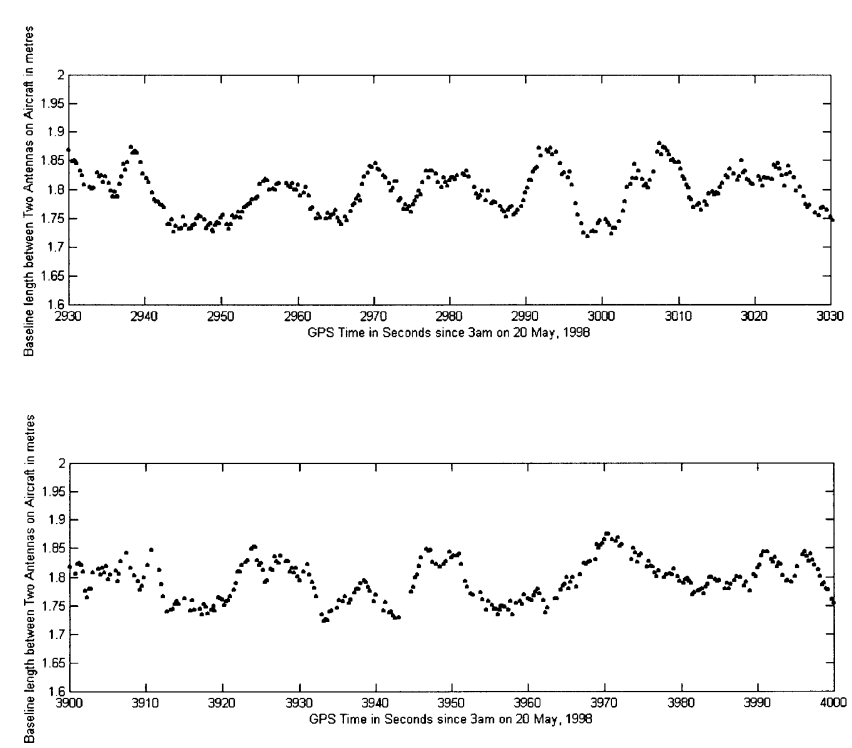

Fig. 10. Distance between two antennas for two runs (the upper figure for the first run, and the lower figure for the second run).

tions, possibly from the LADS laser height noise and the GPS multipath. The multipath effect has been confirmed by the computation of the distance between the two antennas on the aircraft and it appears that the multipath effect is significant for antennas on aircraft. The effect could be of the order of $10 \mathrm{~cm}$ in many cases, and hence multipath mitigation for airborne GPS applications is a challenge.

\section{Conclusions}

The 'ambiguity recovery' technique in combination with the 'linear bias correction' procedure can be used, if the integer ambiguities cannot be resolved correctly. All three brands of GPS receivers tested, the Ashtech Z12, Leica MC1000 and NovAtel Millennium, can be used for longrange, carrier phase-based, airborne applications with high data rate output $(5 \mathrm{~Hz}, 10 \mathrm{~Hz}$ and $4 \mathrm{~Hz}$ ). The discrepancies are at centimetre level among the heights derived from different data sets. Sea surface and bathymetric charts at sub-decimetre level accuracy can be derived from GPS and LADS two-colour laser measurements with a spatial resolution of a few metres. The main error sources appear to be multipath and laser ranging measurement noise.

Acknowledgments. The assistance of the following is acknowledged: Martin Huolohan and Ian MacKay of Leica Geosystems Pty Ltd., Rod McLeod and Justin Legg of SAGEM Australasia (Ashtech), Graeme Hooper of GPSat Systems (NovAtel), David Robertson and Xiujiao Han of UNSW, Ralph Abbot, Dallas Lane and Chris Jones (DSTO), OIC LADS and the RAN crew, the LADS pilots and Rod Kelly with the LADS Corp field team, the Joint Defence Force Facility on Thursday Island and The Bureau of Meteorology at Cairns airport.

\section{References}

Ackermann, F. and H. Schade, Application of GPS for aerial triangulation, Photogrammetric Engineering and Remote Sensing, 59(11), 1625-1632, 1993.

Han, S., Ambiguity recovery for GPS long range kinematic positioning, Navigation, Journal of US The Institute of Navigation, 44(2), 257-266, 1997.

Han, S. and C. Rizos, Centimeter GPS kinematic or rapid static survey without ambiguity resolution, Surveying and Land Information System, 56(3), 143-148, 1996.

Han, S., C. Rizos, and R. Abbot, Sea surface determination using longrange kinematic GPS positioning and Laser Airborne Depth Sounder techniques, Marine Geodesy, 22, 195-203, 1999.

Sinclair, M., Faster, deeper and cheaper airborne laser bathymetry comes of age ready for the new millennium, Hydro International, 3(3), 6-9, 1999.

S. Han (e-mail: SHan@MagellanGPS.com) and C. Rizos (e-mail: c.rizos@unsw.edu.au) 